\title{
PREFERENCIAS DE LOS MEDIOS DE COMUNICACIÓN EN LOS ESTUDIANTES DE ULADECH CATÓLICA - FILIAL PIURA
}

\author{
PREFERENCES IN THE MEDIA AMONG STUDENTS CATHOLIC \\ ULADECH - SUBSIDIARY PIURA
}

Mario Enrique Nizama Reyes ${ }^{1}$

\section{RESUMEN}

La presente investigación tuvo como objetivo determinar los niveles de preferencias de los medios de comunicación entre los estudiantes de ULADECH Católica - Filial Piura, durante el segundo semestre del año académico 2016. La investigación fue descriptiva - cuantitativa, estando la población constituida por los estudiantes regulares de ambos sexos matriculados en ULADECH Católica - Filial Piura durante el semestre 2016-2, tanto a nivel de pregrado como de postgrado. Con una muestra de 200 estudiantes, por un muestreo no probabilístico, por conveniencia, se aplicó una encuesta, cuatro cuestionarios. Los resultados que se obtuvieron fueron: para la preferencia en televisión el mayor puntaje fue para América TV (34\%); mientras que para la preferencia en diarios el mayor porcentaje lo obtuvo el diario La Hora (también con 34\%); para la preferencia en radios hubo porcentajes muy cercanos entre RPP (23\%) y Karibeña (21\%); finalmente para la preferencia en redes sociales el mayor porcentaje lo obtuvo Facebook (70\%). En cuanto a las conclusiones se puede indicar en primer lugar que el liderazgo de América TV fundamentalmente es por su variada programación acorde a los gustos de sus espectadores. En segundo lugar, el liderazgo de La Hora no se debe al precio sino sobretodo a su entendible contenido. En tercer lugar prefieren las radios. En cuarto lugar las redes sociales tienen un líder consolidado que es Facebook, muy por encima de cualquier otra red social actualmente existente.

PALABRAS CLAVE: Medios de comunicación, preferencias, redes sociales.

1 Magister en Ciencias de la Educación. Docente de la Escuela Profesional de Ingeniería de Sistemas de la Universidad Católica Los Ángeles de Chimbote, filial Piura. 


\begin{abstract}
The objective of this research was to determine the levels of communication preferences among the students of ULADECH Católica - Filial Piura, during the second semester of the 2016 academic year. The research was descriptive - quantitative, with the population constituted by the regular students of both sexes enrolled in ULADECH Católica - Filial Piura during the semester 2016 - 2, both at undergraduate and postgraduate levels. With a sample of 200 students, for a non-probabilistic sampling, for convenience, a survey was applied, four questionnaires. The results obtained were: for television preference the highest score was for América TV (34\%); while for newspaper preference the highest percentage was obtained by the newspaper La Hora (also with 34\%); for the preference in radios there were very close percentages between RPP (23\%) and Karibeña (21\%); finally, for preference in social networks, the highest percentage was obtained by Facebook (70\%). Regarding the conclusions, it can be indicated in the first place that the leadership of América TV is basically due to its varied programming according to the tastes of its viewers. Secondly, the leadership of La Hora is not due to the price but above all to its understandable content. Third, they prefer radios. Fourth, social networks have a consolidated leader that is Facebook, far above any other existing social network.
\end{abstract}

KEY WORDS: Media, preferences, social networks.

\title{
INTRODUCCIÓN
}

Los medios de comunicación, de acuerdo a Fischer (2004), son todos aquellos instrumentos tecnológicos utilizados en nuestra actual sociedad con la finalidad de comunicar y/o informar mensajes en medios textuales, sonoros, visuales, audiovisuales o multimedios. Estos mismos instrumentos se pueden utilizar para generar una comunicación de manera masiva, es decir orientarlos a muchos millones de personas, como es el caso de la televisión o la radio, y en otras ocasiones, sólo se pueden orientar para transmitir información a pequeños grupos sociales, como es el caso de los diarios locales o institucionales.

Se puede afirmar que de manera diaria, los individuos, organizaciones y comunidades acceden a diversos materiales para informarse de eventos diversos (políticos, sociales, económicos y culturales), tanto a nivel local como mundial. Con el inicio del presente siglo, y en casi la totalidad del planeta, los diarios, estaciones de televisión y radio y páginas web son ejemplos de la necesidad y compleja naturaleza de los medios de comunicación, convirtiéndose estos en medios fundamentales para el establecimiento y desarrollo de los procesos de interacción humana ya que los medios de comunicación son la representación instrumental de la necesidad de relacionarse entre sí que tienen todos los seres humanos, pues 
mediante ellos se pueden describir, analizar y discutir situaciones y problemas de nuestra realidad además de poder establecer procesos de intercambio de conocimientos y debates de índole social (Fischer, 2004).

Sin lugar a dudas en la sociedad moderna, todos los medios de comunicación son la forma más eficiente, rápida y a veces hasta más económica de transmitir un mensaje. Referenciando a Kotler (2003) se puede ejemplificar que una página web es muy útil para publicar un contenido que necesite divulgación inmediata entre varios sectores de nuestra comunidad, pero estos mismos medios de comunicación pueden convertirse en herramientas de manipulación social, es decir debe entenderse que los de comunicación pueden servir para hacerse escuchar o para imponer una visión de los problemas, pudiendo servir también como un reflejo de un momento específico, como un documento histórico o como un dispositivo en el que se registra lo positivo y lo negativo de una situación o de un contexto cotidiano determinado.

Por el gran avance y revolución tecnológica, incluyendo a los medios de comunicación, como ha sucedido en las últimas décadas desde la aparición de internet, se han desarrollado nuevos medios virtuales de comunicación masiva, que vuelven fácil la transferencia de datos e información de forma globalizada y en tiempo real. Pero el papel fundamental de los medios de comunicación, de acuerdo a Lamb (2002), no sólo va a radicar en el hecho de que sean una herramienta muy útil para realizar una de las necesidades más básicas del ser humano como es la interacción social, sino que además juegan y han jugado un papel primordial en la formación de la Opinión Pública (el Cuarto Poder), llegando incluso a ser relevantes para el fortalecimiento o extinción de algunos gobiernos, por ejemplo como sucedió en nuestro país con el famoso video Kouri - Montesinos que dio inicio al fin del gobierno de Fujimori.

Por todo lo antes expuesto y detallado se justifica el presente trabajo de investigación, pues resulta significativo conocer los niveles de preferencia de nuestros estudiantes (como reflejo de la sociedad piurana) respecto a los medios de comunicación masivos más representativos en la actualidad, planteándose el siguiente problema: ¿Cuáles son los niveles de preferencias de los medios de comunicación entre los estudiantes de ULADECH Católica - Filial Piura, durante el segundo semestre del año académico 2016? Para dar respuesta a este problema, se planteó el siguiente objetivo general: Determinar los niveles de preferencias de los medios de comunicación entre los estudiantes de ULADECH Católica - Filial Piura, durante el segundo semestre del año académico 2016. 


\section{MATERIAL Y MÉTODOS}

En la investigación la población estuvo constituida por los estudiantes regulares de ambos sexos matriculados en ULADECH Católica - Filial Piura durante el semestre 2016 - 2, tanto a nivel de pregrado (de las nueve carreras profesionales) así como de postgrado. La muestra estuvo constituida por 200 estudiantes, determinada a partir de un muestreo no probabilístico, por conveniencia de la investigación.

Se usó el método de "recordación" para medir las preferencias de los medios de comunicación seleccionados, aplicando la técnica de la encuesta y los instrumentos específicos fueron cuatro cuestionarios, uno para cada uno de los medios de comunicación seleccionados: televisión, diarios, radio y redes sociales. Por cada cuestionario se trabajó con dos dimensiones, género (con 2 ítems) y edad (con 4 ítems) tal y como se observa en las tablas anexas de resultados (Semenik, 2004).

En el proceso de la investigación se utilizó el método descriptivo - cuantitativo. Es descriptivo por cuanto se obtuvieron datos y se hicieron mediciones para cada uno de los ítems de los cuatros medios investigados (televisión, diarios, radio y redes sociales); y es cuantitativo por cuanto se han recogido y analizado datos definidos, exactos y precisos de cada uno de esos ítems en las dos dimensiones de evaluación utilizadas (género y edad).

\section{RESULTADOS}

Tabla 1

PREFERENCIA EN TELEVISIÓN

\begin{tabular}{lccccccc}
\hline Ítem & América & ATV & Latina & Paname. & TVPerú & Cable & Total \\
\hline Género & & & & & & & \\
Masculino & $31 \%$ & $14 \%$ & $17 \%$ & $7 \%$ & $3 \%$ & $28 \%$ & $100 \%$ \\
Femenino & $34 \%$ & $23 \%$ & $18 \%$ & $3 \%$ & $2 \%$ & $20 \%$ & $100 \%$ \\
\hline Edad & & & & & & & \\
Menos de 18 & $35 \%$ & $25 \%$ & $12 \%$ & $4 \%$ & $0 \%$ & $24 \%$ & $100 \%$ \\
De 18 a 30 & $34 \%$ & $12 \%$ & $21 \%$ & $4 \%$ & $1 \%$ & $28 \%$ & $100 \%$ \\
De 31 a 45 & $38 \%$ & $10 \%$ & $25 \%$ & $10 \%$ & $5 \%$ & $12 \%$ & $100 \%$ \\
Más de 45 & $30 \%$ & $19 \%$ & $11 \%$ & $8 \%$ & $8 \%$ & $24 \%$ & $100 \%$ \\
\hline Promedio & $34 \%$ & $17 \%$ & $17 \%$ & $6 \%$ & $3 \%$ & $23 \%$ & $100 \%$ \\
\hline
\end{tabular}


Tabla 2

PREFERENCIA EN DIARIOS

\begin{tabular}{lccccccccc}
\hline Ítem & La Hora & Correo & Trome & Tiempo & Repúbl. & Comerc & Popular & Otros & Total \\
\hline Género & & & & & & & & & \\
Masculino & $33 \%$ & $14 \%$ & $14 \%$ & $9 \%$ & $14 \%$ & $8 \%$ & $3 \%$ & $5 \%$ & $100 \%$ \\
Femenino & $35 \%$ & $23 \%$ & $20 \%$ & $9 \%$ & $3 \%$ & $4 \%$ & $4 \%$ & $2 \%$ & $100 \%$ \\
\hline Edad & & & & & & & & & \\
Menos de 18 & $31 \%$ & $14 \%$ & $30 \%$ & $5 \%$ & $3 \%$ & $4 \%$ & $5 \%$ & $8 \%$ & $100 \%$ \\
De 18 a 30 & $39 \%$ & $11 \%$ & $15 \%$ & $8 \%$ & $8 \%$ & $10 \%$ & $7 \%$ & $2 \%$ & $100 \%$ \\
De 31 a 45 & $28 \%$ & $10 \%$ & $14 \%$ & $10 \%$ & $10 \%$ & $12 \%$ & $5 \%$ & $10 \%$ & $100 \%$ \\
Más de 45 & $36 \%$ & $23 \%$ & $11 \%$ & $9 \%$ & $11 \%$ & $6 \%$ & $3 \%$ & $1 \%$ & $100 \%$ \\
\hline Promedio & $34 \%$ & $16 \%$ & $17 \%$ & $8 \%$ & $8 \%$ & $7 \%$ & $5 \%$ & $5 \%$ & $100 \%$ \\
\hline
\end{tabular}

Tabla 3

PREFERENCIA EN RADIOS

\begin{tabular}{lccccccc}
\hline Ítem & RPP & Karibeña & Nueva Q Antena 10 & La 99 & Otros & Total \\
\hline Género & & & & & & & \\
Masculino & $26 \%$ & $22 \%$ & $14 \%$ & $12 \%$ & $12 \%$ & $14 \%$ & $100 \%$ \\
Femenino & $18 \%$ & $23 \%$ & $19 \%$ & $14 \%$ & $8 \%$ & $18 \%$ & $100 \%$ \\
\hline Edad & & & & & & & \\
Menos de 18 & $11 \%$ & $25 \%$ & $20 \%$ & $20 \%$ & $12 \%$ & $12 \%$ & $100 \%$ \\
De 18 a 30 & $15 \%$ & $21 \%$ & $21 \%$ & $14 \%$ & $11 \%$ & $18 \%$ & $100 \%$ \\
De 31 a 45 & $31 \%$ & $19 \%$ & $16 \%$ & $12 \%$ & $14 \%$ & $8 \%$ & $100 \%$ \\
Más de 45 & $34 \%$ & $17 \%$ & $11 \%$ & $20 \%$ & $9 \%$ & $9 \%$ & $100 \%$ \\
\hline Promedio & $23 \%$ & $21 \%$ & $17 \%$ & $15 \%$ & $11 \%$ & $13 \%$ & $100 \%$ \\
\hline
\end{tabular}


Tabla 4

PREFERENCIA EN REDES SOCIALES

\begin{tabular}{lcccc}
\hline Ítem & Facebook & Twitter & Otros & Total \\
\hline $\begin{array}{l}\text { Género } \\
\text { Masculino }\end{array}$ & $68 \%$ & $18 \%$ & $14 \%$ & $100 \%$ \\
Femenino & $74 \%$ & $14 \%$ & $12 \%$ & $100 \%$ \\
\hline Edad & & & & \\
Menos de 18 & $84 \%$ & $10 \%$ & $6 \%$ & $100 \%$ \\
De 18 a 30 & $70 \%$ & $18 \%$ & $12 \%$ & $100 \%$ \\
De 31 a 45 & $66 \%$ & $25 \%$ & $9 \%$ & $100 \%$ \\
Más de 45 & $56 \%$ & $36 \%$ & $8 \%$ & $100 \%$ \\
\hline Promedio & $70 \%$ & $20 \%$ & $10 \%$ & $100 \%$ \\
\hline
\end{tabular}

Los resultados del presente trabajo de investigación se han agrupado en cuatro tablas, una tabla de preferencias por cada uno de los cuatros medios de comunicación estudiados en el presente trabajo de investigación. En cada tabla se registran dos dimensiones, una dimensión respecto a las preferencias según género ( 2 ítems) y otra dimensión que refleja las preferencias según la edad (4 ítems).

En las preferencias registradas respecto a los canales de televisión (Tabla 1: Preferencias en televisión) para la dimensión de género, ítem masculino, el valor más alto de 31\% indica una preferencia por América TV, siendo el más bajo valor de 3\% para TV Perú. En la misma dimensión de género, pero para el ítem femenino, la mayor preferencia se registra también para América TV y el valor más bajo igualmente para TVPerú. En cuanto a la dimensión edad, para el primer ítem, menos de 18 años, el mayor valor se registra para América TV con 35\%, seguido de ATV con 25\% y cierra TVPerú con 0\%. En el ítem de 18 a 30 años se sigue la tendencia en el sentido que América TV registra el mayor porcentaje con un 34\% y el menor valor en este ítem también es para TVPerú con un apenas un 1\%. En lo referente al ítem de 31 a 45 años América TV sigue con el valor más alto equivalente al 38\% y también lo cierre TVPerú con el valor más bajo igual al 5\%. Finalmente para el ítem de más de 45 años también lo lidera América TV con un 30\%, pero hay un empate en los valores más bajos entre Panamericana y TVPerú cada 
uno con $8 \%$ respectivamente. Como resultado global para esta primera tabla referente a la televisión, y tomando como referencia el promedio de los resultados se pudo observar que la mayor preferencia la tiene América TV (34\%) y la menor preferencia TVPerú (sólo un 3\%).

Los resultados obtenidos para los diarios (Tabla 2: Preferencia en diarios), muestra que en la dimensión de género, el ítem masculino tiene su mayor valor de preferencia para el diario La Hora con 33\% al igual que para el ítem femenino con un $35 \%$. Para la edad la tendencia se mantiene, es decir el diario La Hora se mantiene con los más altos valores para cada uno de los cuatro rangos de edades definidos. A nivel global para esta segunda tabla, y tomando siempre como referencia el promedio de los resultados, se observó que el mayor resultado lo tuvo La Hora (34\%), seguido del diario Trome (17\%), cerrando con el diario El Popular (5\%).

En el caso de las preferencias de las emisoras de radio (Tabla 3: Preferencia en radios), el género masculino tiene su valor porcentual más alto para la radio RPP con un 26\%, y el valor más bajo para la radio Antena 10 y La 99 cada una con un $12 \%$. En el género femenino, el valor más alto es para radio la Karibeña (23\%). Para la dimensión edad, los resultados si se presentan diferentes, pues para los ítems de menos de 18 años y de 18 a 30 años sus valores más altos son para radio La Karibeña, mientras que para los ítems de 34 a 45 años y más de 45 años los valores más altos son para RPP. Así de manera global, en esta tercera tabla, en promedio el mayor porcentaje es para RPP (23\%), seguido de La Karibeña (21\%) y terminando con el menor porcentaje para radio La 99 (11\%).

Finalmente, en lo referido a las tan omnipresentes redes sociales (Tabla 4: Preferencia en redes sociales), tanto para el género masculino como femenino se pudieron observar los valores más altos para Facebook (68\% y 74\% respectivamente), tendencia que también se mantuvo en cada uno de los cuatro ítems de la dimensión edad. A partir de los promedios de los resultados se obtuvo a Facebook con el valor porcentual más alto (70\%), seguido de Twitter (20\%), cerrando la categoría de otras redes sociales $(10 \%)$.

\section{DISCUSIÓN}

$\mathrm{Al}$ analizar los resultados de la presente investigación aplicada entre nuestros estudiantes, que como se reitera es un reflejo de nuestra sociedad piurana por cuanto en nuestra Universidad confluyen ciudadanos de todos los niveles, estratos y realidades locales y nacionales, se ha podido observar tendencias bastantes 
marcadas con respecto a las preferencias de los cuatro medios de comunicación seleccionados para el presente estudio.

Empezando por la preferencia referida a la televisión el liderazgo es claramente para América TV, registrándose una mayor preferencia por parte del género femenino ( $3 \%$ más que el género masculino) básicamente por el tema de su programación (telenovelas, series, producción nacional y sobretodo los "reality" juveniles). Respecto a la edad, el liderazgo de preferencia igual se mantiene en los cuatro rangos definidos, por la misma razón antes mencionada, es decir la programación que ofrece. Con la mitad de la preferencia que el líder América TV encontramos a ATV y Latina, quienes también tienen una variada y aceptada programación, no sucediendo lo mismo con Panamericana y TVPerú (canal estatal) cuya programación actual no ha llegado a ser de la preferencia de los televidentes (hablando netamente de gustos y no necesariamente de calidad de los contenidos como es el caso de los programas culturales de TVPerú). Mención aparte merece la televisión por cable, que en su conjunto posee casi la cuarta parte entre las preferencia de los televidentes encuestados, aunque no llega a superar al canal nacional líder, primordialmente por cuanto la programación de este tipo de televisión es poco variada o incluso repetitiva y monótona (canales sólo de películas, canales sólo de música, canales sólo de deportes, etc.).

En lo referido a las preferencias de los diarios, igual que con la televisión hay un líder definido tanto en la dimensión de género como de edad que es el diario La Hora, sustancialmente por su diagramación sencilla y seria a la vez, además de poseer un contenido periodístico entendible para sus lectores, es decir fácil de leer para la mayoría de la gente. El factor precio no ha tenido una intervención significativa, pues casi todos los diarios referidos (excepto La República y El Comercio) tienen un costo de 70 céntimos, sin embargo el promedio porcentual entre el líder (La Hora con 34\%) y el segundo en preferencia (Trome con 17\%) como se ha observado es del doble a pesar de que entre ambos la diferencia del costo sólo es de 20 céntimos. Cabe resaltar que el diario líder, es netamente local.

En cuanto a la preferencia en radios, aquí si hay una mayor disparidad entre las preferencias de acuerdo a las dimensiones analizadas. Si bien es claro que el mayor porcentaje de preferencia lo tiene RPP, también es claro que no hay una marcada ventajas con el segundo lugar (apenas $2 \%$ en promedio) como sucedía con las preferencias de la televisión y los diarios. Sólo al analizar las preferencias en la dimensión género ya se marca una diferencia entre el género masculino que 
prefiere a RPP (noticias con 26\%) y el género femenino que prefiere radio Karibeña (música tropical con 23\%). En la dimensión edad, los ítems de menos de 18 años y de 18 a 30 años muestran una preferencia también a la Karibeña (que como ya se mencionó mayormente ofrece música de ritmo tropical), mientras que los ítems de 31 a 45 años y de más de 45 años mostraron una preferencia hacia RPP (que como también ya se mencionó es una cadena de noticias de gran prestigio y credibilidad).

Finalmente en lo que se refiere a las preferencias en redes sociales también hubo un líder indiscutible, Facebook con $70 \%$ muy por encima del Twitter con sólo 20\%. Este liderazgo absoluto de Facebook se reflejó tanto en los dos ítems del género, así como en los cuatro ítems de la edad. Esta marcada y amplia diferencia se da principalmente por la versatilidad de Facebook en conectar a personas de todas partes del planeta, y que muchos utilizan para reencontrarse con viejos amigos, con gente a la que no han visto por mucho tiempo, así como las opciones que ofrece para crear grupos con diversos criterios, compartir y comentar fotografías y todo tipo de archivos multimedia, participar de encuestas y enviar mensajes.

\section{CONCLUSIONES}

En base a todos los resultados que se han podido presentar, sistematizar y discutir previamente, como conclusiones del presente trabajo podemos mencionar las siguientes:

- El liderazgo de las preferencias en televisión indiscutiblemente es para América Televisión, tanto a nivel de género como de edad, preferencia basada fundamentalmente por la variedad de su programación que sintoniza con los gustos de los televidentes.

- Para los diarios, también hubo un liderazgo de preferencia marcado para La Hora principalmente por su diagramación sencilla y su contenido entendible para sus lectores.

- La preferencia en radios fue la más dispersa, pues si bien es cierto hubo una emisora radial con mayor puntaje, este porcentaje de preferencia no fue muy alto ni definido con respecto al segundo lugar, tal es así que hubo ítems donde se alternaba el liderazgo de preferencia entre el primer lugar en promedio (RPP) y el segundo lugar en promedio (Karibeña). 
- En las redes sociales la preferencia fue marcada y altamente definida para Facebook, muy por encima de Twitter y de otras redes sociales (como Youtube, Instagram o Google+) primordialmente por su versatilidad e integración de servicios.

\section{REFERENCIAS CONSULTADAS}

Fischer, L. (2004). Mercadotecnia. Madrid, España: Mc Graw Hill.

Kotler, P. (2003). Fundamentos de Marketing. México, México: Prentice Hall.

Lamb, C. (2002). Marketing. México, México: International Thomson Editores.

Semenik, R. (2004). Publicidad. México, México: International Thomson Editores. 